\title{
BNIMS: Block-based Non-iterative Mean-shift Segmentation algorithm for Medical Images
}

\author{
P. Pedda Sadhu Naik ${ }^{1^{*}}$, T. Venu Gopal ${ }^{2}$ \\ ${ }^{1}$ CSE Department, JNTUK, Kakinada, India \\ ${ }^{2}$ CSE Department, JNTUHCE, Sulthanpur, Medak, India \\ *Corresponding author, email: ppsnaik@gmail.com, t_vgopal@ rediffmail.com
}

\begin{abstract}
This paper proposed a novel Block based Mean Shift Image Segmentation Algorithm to significantly reduce the computation and improve the segmentation accuracy for high resolution Medical Image. One of the challenging tasks in the image analysis and computer vision area is to correctly classify the pixels as there are no crisp borders among entities in an image. In this proposed methodology, it is observed that the computational complexity of the procedure is diminished by combining the pixels of an image of size MXN into non overlapping image blocks of size $3 \times 3$ by eliminating the iterative way of the mean shift procedure. This proposed algorithm shrinks the size of the image by one third of its original image for the computational purpose and then equalizes the number of computations for each new image pixel by constructing links between pixels using their first mean-shift vectors without any iteration process. The accurateness and effectiveness of the proposed methodology is matched with the existing Iterative Mean Shift Algorithm by accomplishing the empherical experiments on the Medical Images (Pathologies Buccales and Eye Retina) composed along with the similarity measures.
\end{abstract}

Keywords: Image Segmentation, Mean-shift, medical images, Computational Complexity

Copyright (C) 2016 APTIKOM - All rights reserved.

\section{Introduction}

Image segmentation is one of the important extensive way of classifying the pixel values of an image appropriately in decision oriented applications. It partitions an image into even and nonoverlapping are as depending on some similarity measure. It is one of the most challenging tasks in image processing and a very important pre-processing step in the fields of computer vision, image analysis, pattern recognition, medical image processing, remote sensing and geographical information system. Image Segmentation is broadly used by customer display schemes to improve the eminence of image processing techniques. Compared to a monochrome image, a color image carries much more information [1]. Thus, color image segmentation is becoming increasingly prevalent nowadays. Image segmentation has two elementary properties of image. They are 1) intensity values comprising of discontinuity that denotes the immediate or unexpected variations in intensity as boundaries and 2) similarity that denotes to segregate a digital image into areas according to some pre-specified similarity principle.

Image Segmentation is a frequently used low level image processing methodology that categorizes the image. The clustering techniques are employed to categorize the identical regions depending on color features. The mean shift is an entrenched and dominant non-parametric clustering technique. It is a prominent choice for image segmentation because of its non-parametric environment and nominal customer input. The information in the image are conserved due to non-parametric characteristics of exploration which does not consider any specific arrangement of data. The mean shift technique established on image segmentation is a straightforward extension of the disjointedness conserving smoothing algorithm. The most important property of the image segmentation algorithm is its stability which is derived significantly from Mean Shift Algorithm. The traditional mean shift procedure was proposed by Fukunaga and Hostetler in 1975 and reinstated by Cheng [2] in 1995. According to Cheng, the mean shift is a simple iterative technique that moves every data point to a fixed size dynamic window to the mean of the data points in its neighborhood. The change is given by considering the gradient of the kernel density approximation that constantly points towards the direction of the maximum rise in density. 
There are some of the prevailing Mean Shift rendering Segmentation Algorithm that are introduced by adapting diverse methods of density approximations. The mean shift does not work well in higher dimension since the number of local maxima is pretty high and it may converge to local optima quickly. The computational complexity of the standard iterative mean shift rendering image segmentation algorithm is $\mathrm{O}\left(\mathrm{kN}^{2} \mathrm{D}\right)$ where $\mathrm{k}$ is the number of iterations, $\mathrm{N}$ is the number of pixels and $\mathrm{D}$ is a data dimension [3]. This paper is an extended version of image segmentation using linked mean shift [4] that matches the number of computations for every pixel by constructing links between pixels using their first mean-shift vectors without iteration process.

Thus, to overcome the limitation of the standard Mean Shift algorithm by using the non-iterative procedure and further minimizes the computational complexity of the image segmentation using linked mean shift vector a novel methodology is suggested. Block based Non-Iterative Mean Shift (BNIMS) segmentation algorithm proposed to significantly reduces the computational complexity and improve the segmentation accuracy for High resolution Medical Images. The main idea of this novel methodology is to group the original image of size MXN into blocks of size $3 \times 3$ by considering the center of the block or by considering the average of the pixels in the block as the new pixel value for the image. This process reduces the computational complexity of the proposed methodology to a fraction of a one-ninth part of the standard mean shift algorithms complexity that is $\mathrm{O}\left(\mathrm{k} \frac{N^{2}}{9} D\right)$.

A brief introduction is given to the mean shift based image segmentations algorithm along with the motivation for the proposed methodology in this section. The Section 2 discusses about the existing methodologies in Mean Shift based Image Segmentation. The proposed Methodology is briefly discussed in the Section 3. The experimentation results and its brief analysis is given in Section 4. Finally, Section 5 concludes the proposed novel methodology with the reduced computational complexity.

\section{Existing Methodologies}

A large amount of importance has been created recently in the areas of image analysis and is becoming furthermore dominating. In this perspective, diversity of segmentation procedures have been investigated in the literature comprising of Histogram Thresholding [5], Level Set Methods [6, 7], GraphBased Approaches [8-11], Fuzzy Logic techniques [12]. For example, image segmentation is widely used to improve the quality of 2D-to-3D conversion [13], image in-painting [14], motion estimation [15], and stereo matching [16].

The mean shift was initially established by Fukunaga and Hostetler [17] depending on kernel density approximation and latter functioned on numerous computer vision tasks such as tracking [18-20], image segmentation [21, 22], information fusion [23], clustering and classification [24, 25] and video processing [26].Comaniciu [21]discovered the significance of the various components of the gradient, $\widehat{\nabla} \mathrm{f}(\mathrm{x})$ of the kernel density estimate (k.d.e.).Supposing that $\mathrm{x}_{\mathrm{i}}$ corresponds to any point in the neighborhood of size with a kernel band width and considering a Gaussian kernel $g$ (), the gradient of the kernel density approximation can be expressed as the product of two terms.

In equation 1, the first term is the K.D.E. at the point $\mathrm{x}$. The second displacement term is called the mean shift vector, $m(x)$. The gradient of the K.D.E. $\nabla^{\wedge} \mathrm{f}(\mathrm{x})$ is proportional to the K.D.E. by a factor given by the mean shift vector, $\mathrm{m}(\mathrm{x})$. The first part of $\mathrm{m}(\mathrm{x})$ relates to the calculated neighborhood center of mass, C.A proof of the convergence of the mean shift technique is given in [27]. The mean shift technique is influences in high density areas, as in case of multidimensional data sets evidences to be computationally expensive. Recently, [28, 29, 30, 31] work has been done to achieve fast mean shift methods capable of processing multidimensional data sets easily.

A unique path assigned mean shift algorithm was applied for color segmentation that adapts an iterative process that searches for the mode value for every pixel and clusters the final modes for the segmentation [32]. De Menthon [29] presented a hierarchical mean shift method. A hierarchical clustering technique was employed by recurrently applying the mean shift over progressively large bandwidth, with every stage employing the outcomes of the former to initialize. Zhang [31] presented an exclusive fast mean shift model and functioned it on color segmentation. A well-known Mean Shift algorithm is derived to build a proven stable algorithm, for which [21] propose a tile-wise segmentation method of theory guarantees to get strict identical results with respect to whole-at-once processing.

A brief outline of the mean shift algorithm is presented as follows. Assumed a group of data points $\{x \mid i=1,2, \ldots \ldots, N\}$ in the d-dimensional feature space, the kernel density estimatorwith symmetric kernel function $\mathrm{K}($.$) and fixed bandwidth \mathrm{h}$ can be given as [33] 


$$
f_{k}(x)=\frac{1}{N} \sum_{i=1}^{N} \frac{1}{h^{d}} k\left(\left\|\frac{x-x_{i}}{h}\right\|^{2}\right)
$$

Where $\mathrm{k}($.$) is the profile of kernel \mathrm{K}$ such that $\mathrm{K}(\mathrm{x})=\mathrm{ck}\left(\|x\|^{2}\right)$ and $\mathrm{c}$ is a normalization constant. When the derivative of $\mathrm{k}\left(\right.$.) prevails, $\mathrm{g}()=.-k^{\mid}($.$) can be used asa profile to describe a new$ kernel $\mathrm{G}(\mathrm{x})$ such that $G(x)=c^{\mid} g\left(\|x\|^{2}\right)$ with normalization constant c. considering the gradient of (1) we attain

$$
m(x)=C \frac{\widehat{\nabla} f_{K}(x)}{\widehat{f_{G}(x)}}
$$

Where

$$
m(x)=\frac{\sum_{i=1}^{N} \frac{1}{h^{d+2}} x_{i} g\left(\left\|\frac{x-x_{i}}{h}\right\|^{2}\right)}{\sum_{i=1}^{N} \frac{1}{h^{d+2}} g\left(\left\|\frac{x-x_{i}}{h}\right\|^{2}\right)}
$$

is called the mean shift vector, and $\mathrm{C}$ is a constant. From (2) we observe that $\mathrm{m}(\mathrm{x})$ points towards the sharpest ascent direction of the density function $\widehat{f_{K}(x)}$ thus the mean shift iteration is a

$$
x^{(k+1)}=x^{k}+m\left(x^{k}\right), \quad k=1,2, \ldots \ldots
$$

Hill climbing procedure to the nearest maximum of $f_{K}(x)$

\section{Proposed Methodology}

The Standard Iterative Mean Shift based Image Segmentation Algorithm although provides accurate and consistent results, it suffers from the critical disadvantage of intensive computational complexity $\mathrm{O}\left(k N^{2} D\right)$. Owing to its tremendous complexity, the algorithm functions extremely slow, thus, it is necessary to accelerate the algorithm. One way to accelerate the Standard algorithm is to reduce the computational complexity of the algorithm and to make it suitable for high resolution Medical Images. The proposed novel Methodology is names as the Block based Non Iterative Mean Shift (BNIMS) Segmentation Algorithm.

In this methodology, the selected high resolution medical image is pre-processed before employing a Non Iterative Mean Shift Procedure on the processed image. The pre-processing is achieved by dividing the original image of size MXN into non-overlapping blocks of size 3X3 which considers the mean or the average of the pixels in the block as the new pixel value for the processed image. Then the first mean shift is performed on every pixel of the processed image only once. Here the segmentation is performed on the blocks of the image instead of its pixels which reduced the computational complexity to accomplish the mean shift technique. In the standard mean-shift algorithm, every pixel travels along the mean-shift vector until the vector is converged. In this procedure, the pixels on the traveling path have the same mode [3]. Using this principle [4] an image segmentation algorithm uses linked mean-shift vectors. In this method, the mean-shift vector is computed for each pixel just one time, each pixel is linked to the closest pixel from its mean-shift vector. Then each pixel travels along the link until the link indicates itself. Finally, the last node is regarded as their mode. Figure 1 represents the flow chart for the proposed methodology. 


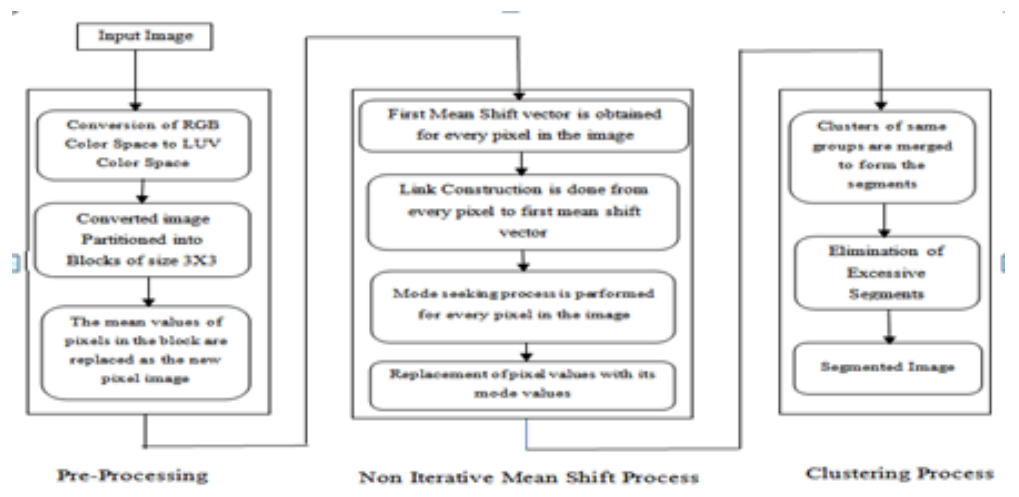

Figure 1. Flow Chart of Block based Non Iterativr Image Segmentation Algorithm

\subsection{Block based Non Iterative Mean Shift Image Segmentation Algorithm:}

a. Initially the color space of high resolution image is converted from RGB color space to the LUV color Space. It is important to select a color space whose Euclidean distance is linearly proportionate to the perceived color difference which is obtained by LUV color space. Thus, the $\mathrm{L}^{*} \mathrm{u}^{*} \mathrm{~V}^{*}$ color space is used instead of the RGB color space.

b. The pre-processing is done on the obtained LUV color space by partitioning the given image into non-overlapping blocks of size $3 \mathrm{X} 3$ and the diminished new image is obtained by replacing the mean of all the pixel values in every blocks as image new pixels values.

c. Now the obtained novel image at any random starting point, a mean shift vector is computed for every pixel by using the equation

$$
y_{i, j+1}=\frac{\sum_{i=1}^{n} y_{i, j} g\left(\left\|\left(y_{i, j}{ }^{r}-x_{i}{ }^{r}\right) / h_{r}\right\|^{2}\right) \cdot g\left(\left\|\left(y_{i, j}{ }^{s}-x_{i}{ }^{s}\right) / h_{s}\right\|^{2}\right)}{\sum_{i=1}^{n} g\left(\left\|\left(y_{i, j}{ }^{r}-x_{i}{ }^{r}\right) / h_{r}\right\|^{2}\right) \cdot g\left(\left\|\left(y_{i, j}{ }^{s}-x_{i}{ }^{s}\right) / h_{s}\right\|^{2}\right)}
$$

Where mean-shift vectors are computed with two independent bandwidths; spatial bandwidth

$\left(h_{s}\right)$ and range bandwidth $\left(h_{r}\right)$. Spatial bandwidth denotes the size of a processing window for spatial domain and range bandwidth means the range of the pixel value.

d. A link is constructed for each pixel. The closest pixel to the mean-shift vector is chosen as the linked pixel. Spatial component, which is the location of a pixel, is used to construct the links since the mean-shift vectors are always generated in ways that the density of its range component increases [3].

e. Then the mode seeking procedure is executed to identify the approach of pixels. For each pixel, the link is traced until it indicates itself. This point is considered as the mode for all connected pixels. Then, the pixel value, which is the range component, is replaced with the range component of its mode; $\mathrm{z}_{\mathrm{i}}=\left(\mathrm{x}_{\mathrm{is}}, \mathrm{y}_{\mathrm{ir}}\right)$. Then, the range component of each block is replaced with the range component of its mean shift mode.

f. Consequently, blocks of the same mode have the same range component, which means the blocks are grouped into the same cluster. Finally a merging process is performed (pre-processing) to merge the neighborhood clusters whose range difference is less than $h_{r} / 2$ [34]. Then the clusters which have the number of pixels less than predefined number $\mathrm{M}$ (pre-defined number) combined to the closest cluster.

\subsection{Advantages of the Proposed Methodology}

a. Decreases the Computational Complexity of the Algorithm to $\mathrm{O}\left(\mathrm{k} \frac{N^{2}}{9} D\right)$.

b. Removes the Iterative process of calculating the mean shift procedure until its convergence.

c. The total number of pixels in the original image are minimized to a factor of N/9 by partitioning into blocks.

d. Minimizes the accuracy degradation due to Non Iterative Process 


\section{Experimental Results and Analysis}

The Experimental Analysis of the proposed Block based Non Iterative Mean Shift (BNIMS) Segmentation Algorithm is evaluated with the help of two types of images in this paper. They are pathologies buccales and Eye retina images containing tumors. The performance evaluation of the proposed BNIMS methodology is compared with existing iterative based traditional mean shift segmentation algorithm.

In the proposed methodology, an RGB image is considered for the experiment which is converted into its LUV color image where the Euclidean Distance is proportionate to the perceived color difference. Then the converted image is divided into blocks of size $3 \times 3$ to perform the segmentation using the proposed and the existing methodology.

Consider the pathologies buccales image as shown in Figure 2 as the original image or input to the proposed methodology and Figure 3 represents the converted LUV color space image. The segmentation of the input image using the traditional iterative mean shift algorithm is shown in Figure 4 along with its color distribution for input and output. The segmentation results of the proposed methodology is shown in Figure 5 with the block size of $3 \times 3$. Different clusters obtained from block division is shown in Figure 6.

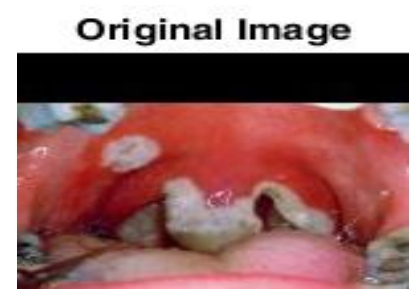

Figure 2. Input Image of pathologies buccales image

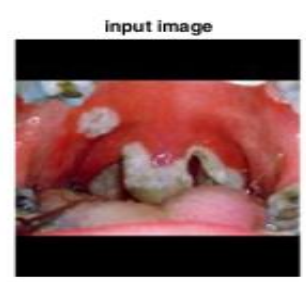

color distribution of input image

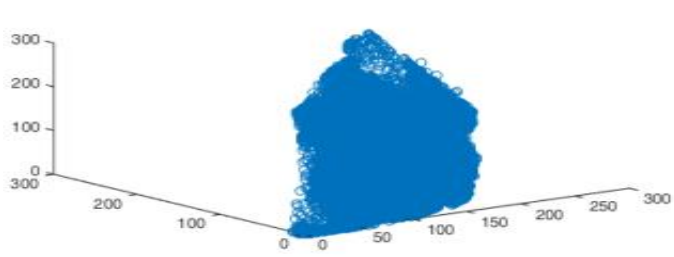

\section{Luv Color Image}

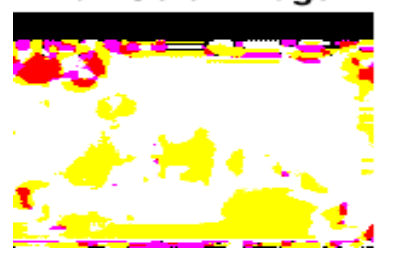

Figure 3. Converted LUV color Space image
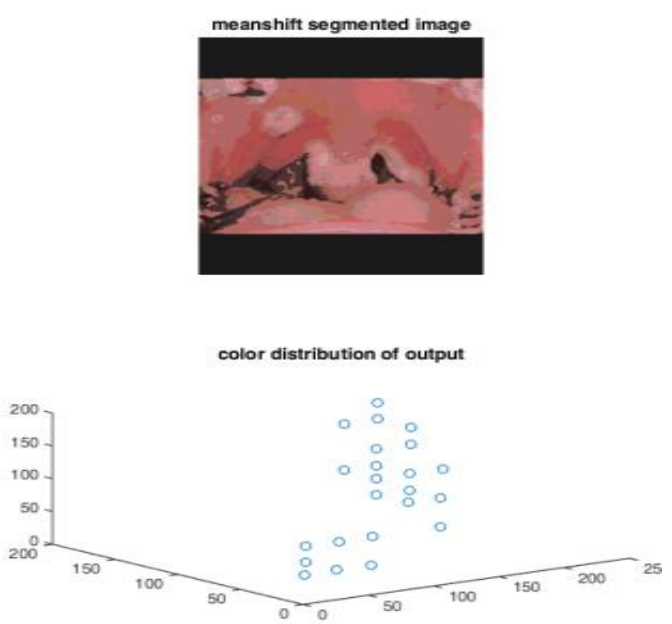

Figure 4. Segmented Image using Traditional Iterative Mean Shift Segmentation Algorithm with its color Distribution of input and output image 


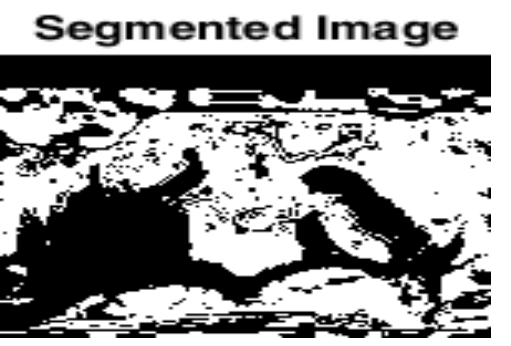

Figure 5. Segmented Image using the Proposed BNIMS Segmentation algorithm

Figure 6. Different Clusters of the image of Block division size 3x3

Comparing the resultant image of Figure 4 and Figure 5 it is clearly observed that there a great difference in the performance of segmentation for the existing and proposed methodology with every minute area segmented clearly in the proposed methodology. It is also observed that there is minute change in the existing iterative method for the resultant image of Figure 3 to the input image Figure 5 as in when compared the proposed non iterative method.

Consider the another pathologies buccales image containing tumor as shown in Figure 7 as the original image or input to the proposed methodology and Figure 8 represents the converted LUV color space image. The segmentation of the input image using the traditional iterative mean shift algorithm is shown in Figure 9 along with its color distribution for input and output. The segmentation results of the proposed methodology is shown in Figure 10 with the block size of $3 x 3$.

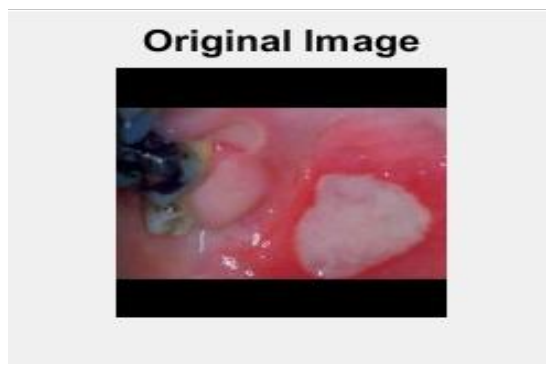

Figure 7. Input Image of pathologies buccales

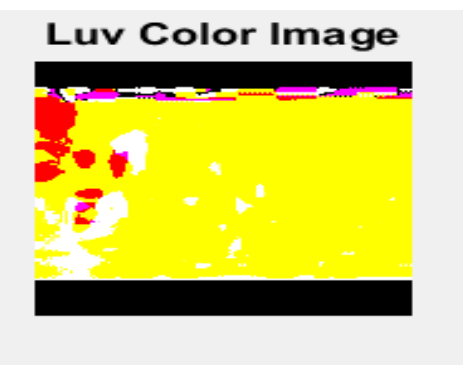

Figure 8. Converted LUV color Space image 


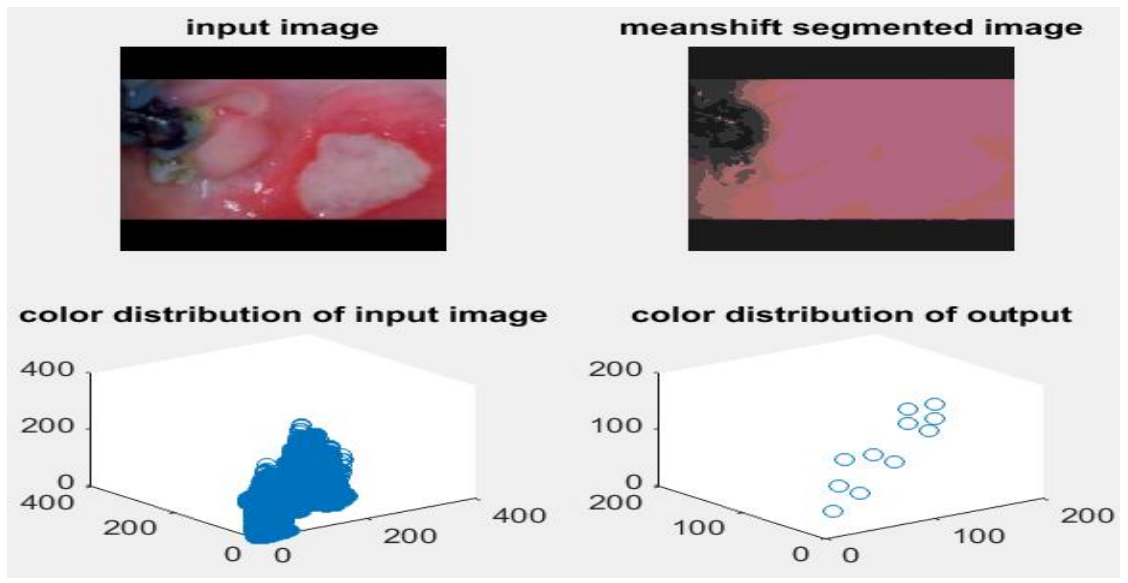

Figure 9. Segmented Image using Traditional Iterative Mean Shift Segmentation Algorithm with its color Distribution of the input and output image

Comparing the resultant image of Figure 9 and Figure 10, it is clearly observed that there a great difference in the performance of segmentation for the existing and proposed methodology with every minute area segmented clearly in the proposed methodology. It is also observed that there is minute change in the existing iterative method for the resultant image of Figure 9 to the input image Figure 10 as in when compared the proposed non iterative method.

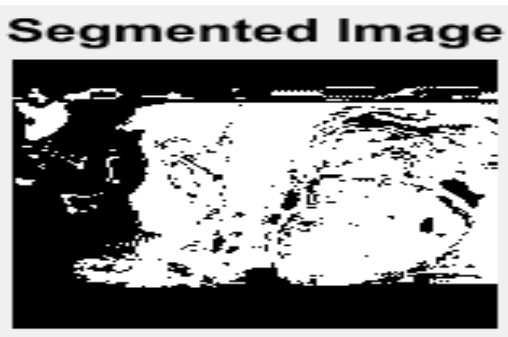

Figure 10. Segmented Image using Proposed BNIMS Segmentation algorithm

Consider the Eye Retina image as shown in Figure 11 as the original image or input to the proposed methodology and Figure 12 represents the converted LUV color space image. The segmentation of the input image using the traditional iterative mean shift algorithm is shown in Figure 13 along with its color distribution for input and output. The segmentation results of the proposed methodology is shown in Figure 14 with the block size of $3 \times 3$.

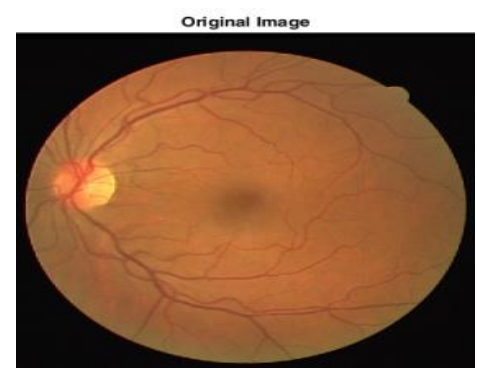

Figure 11. Input image of Eye Retina image

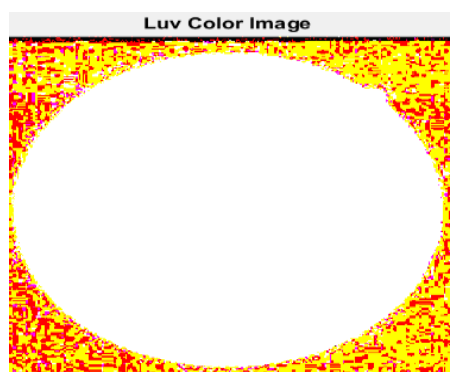

Figure 12. Converted LUV color Space image

APTIKOM J. CSIT Vol. 1, No. 2, 2016 : 46-56 


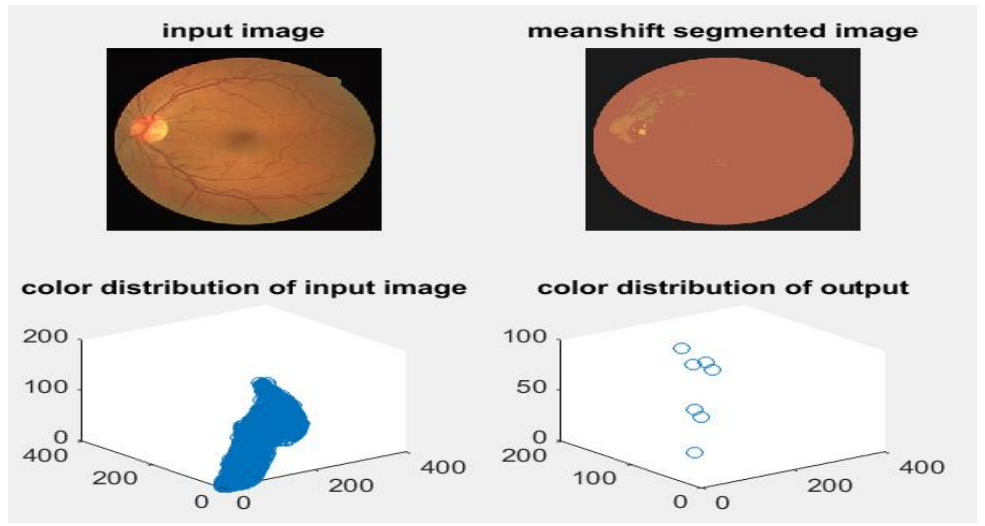

Figure 13. Input Eye Retina Image and Segmented Image using Traditional Iterative Mean Shift Segmentation Algorithm along with its color Distribution of input and output image

Comparing the resultant image of Figure 13 and Figure 14, it is clearly observed that there a great difference in the performance of segmentation for the existing and proposed methodology with every minute area segmented clearly in the proposed methodology. It is also observed that there is minute change in the existing iterative method for the resultant image of Figure 13 to the input image Figure 14 as in when compared the proposed non iterative method.

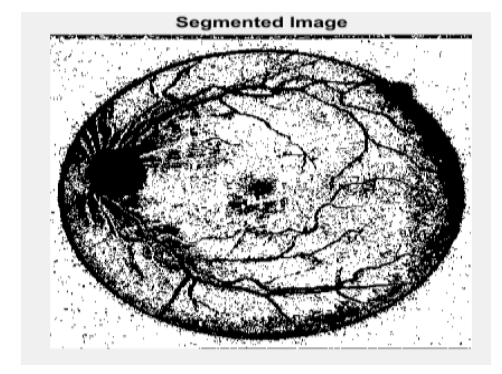

Figure 14. Segmented Image using Proposed BNIMS Segmentation algorithm

In the proposed novel methodology, the author also tried to show that not only for Block Division of Size $3 \times 3$, but the Block sizes of $2 \times 2,4 \times 4$, and $5 \times 5$ also the proposed Block based Non Iterative Mean Shift Segmentation Algorithm gives significant results as in when compared to existing traditional iterative mean shift algorithm.

Consider the pathologies buccales image as shown in Figure 2 as the input where Figure 16 represents different clusters obtained from block division of size $2 \times 2$ with its segmented image with the proposed novel methodology shown in Figure 15. The Figure 18 represents different clusters obtained from block division of size $4 \times 4$ with its segmented image with the proposed novel methodology shown in Figure 17. The Figure 20 represents different clusters obtained from block division of size $5 \times 5$ with its segmented image with the proposed novel methodology shown in Figure 19. It is clearly shown from the resultant image that the proposed novel algorithm also works well for the block division of any size until the complexity of the proposed algorithm remains as $\mathrm{O}\left(\mathrm{k} \frac{N^{2}}{9} D\right)$. 


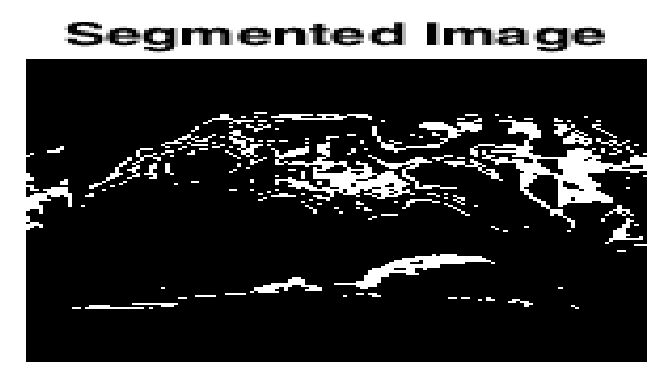

Figure 15. Segmented Image using proposed

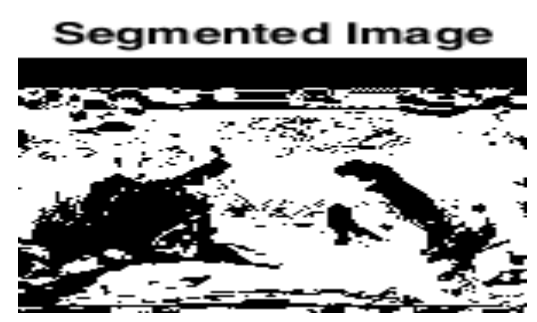

Figure 17. Segmented Image using proposed

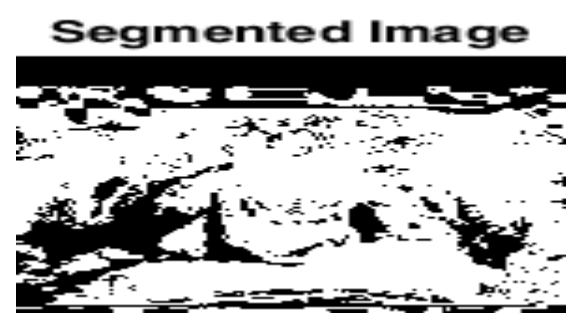

Figure 19. Segmented Image using proposed

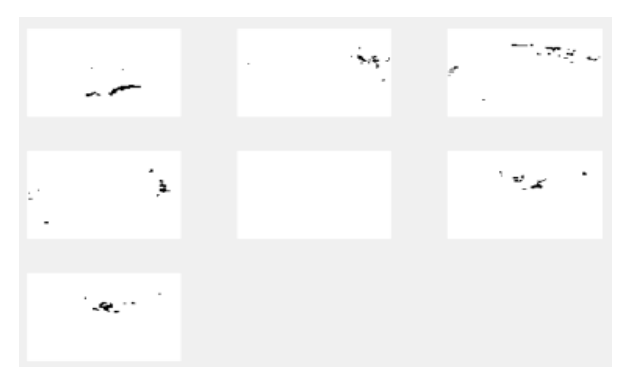

Figure 16. Different Clusters of Input Image Methodology with Block Division of size 2x2

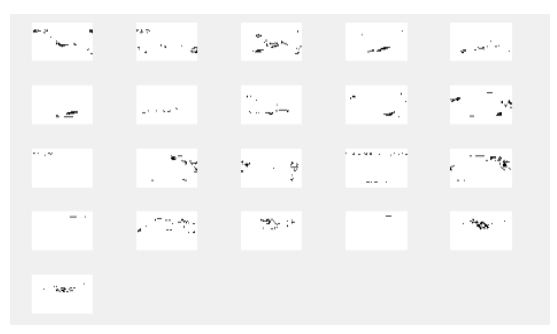

Figure 18. Different Clusters of Input Image Methodology with Block Division of size $4 \times 4$

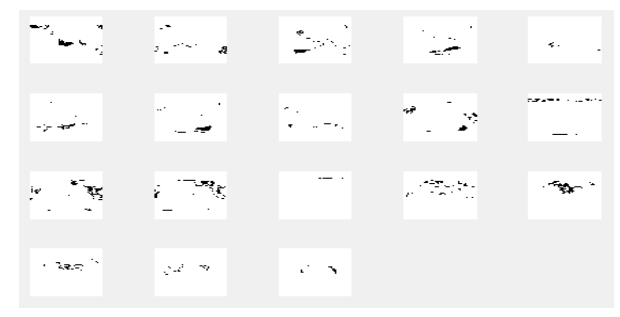

Figure 20. Different Clusters of Input Image Methodology with Block Division of size $5 \times 5$

\section{Conclusion}

The proposed Block based Non Iterative Mean Shift (BPAMS) Image Segmentation Algorithm offers reduces the computational complexity of $\mathrm{O}\left(\mathrm{k} \frac{N^{2}}{9} D\right)$ and improved the segmentation accuracy for high resolution Medical Image Datasets. By constructing the Blocks of size $3 \mathrm{X} 3$ and by linking the pixels using its first mean-shift vector, the proposed algorithm equalizes the number of computations for every pixel reducing the number of computations. Differences in the performance of the proposed algorithm and the existing system are tested using the experimental results and its analysis.

\section{References}

[1] H. D. Cheng, X. H. Jiang, Y. Sun, and J. Wang, "Color image segmentation: advances and prospects," Pattern Recognition, vol. 34, no.12, pp. 2259-2281, 2001.

[2] Yizong Cheng, "Mean shift, mode seeking, and clustering", IEEE Trans. Pattern Anal. Mach. Intell., vol. 17, no. 8, pp.790-799, 1995.

[3] Carreira-Perpinan, Miguel A, “Acceleration strategies for Gaussian mean-shift image segmentation," Computer Vision and Pattern Recognition, 2006 IEEE Computer Society Conference, vol. 1, pp. 1160-1167, June 2006.

[4] H. Cho, S. I. Cho, Y. H. Kim, "Image segmentation using linked mean shift vectors for SIMD architecture," in Proc. IEEE int. Conf.Consumer Electronics, Las Vegas, USA, pp. 484-485, Jan. 2014. 
[5] L. Shafarenko, M. Petrou, and J. V. Kittler, "Histogram based segmentation in a perceptually uniform color space," IEEE Trans. Image Process, vol. 7, no. 9, pp. 1354-1358, 1998.

[6] C. Li, C. Y. Kao, J. C. Gore, and Z. H. Ding, "Minimization of region scalable fitting energy for image segmentation," IEEE Trans. Image Process, vol. 17, no. 10, pp. 1940-1949, 2008.

[7] C. Li, C. Xu, C. Gui, and M. D. Fox, "Level set evolution without re-initialization: A new variation formulation," in Proc. IEEE Conf. Computer Vision and Pattern Recognition, San Diego, USA, 2005, pp.430-436.

[8] Y. Boykov, and M. P. Jolly, "Interactive graph cuts for optimal boundary and region segmentation of objects in $N$-D images," in ICCV, Vancouver, Canada, 2001, pp. 105-112.

[9] Y. Boykov, and G. Funka-Lea, "Graph cuts and efficient n-d image segmentation," International Journal of computer vision, vol. 70, no. 2,pp. 109-131, 2006.

[10] L. Grady, and E. L. Schwartz, "Isoperimetric graph partitioning for image segmentation," IEEE Transaction on Pattern Analysis and Machine Intelligence, vol. 28, no. 3, pp. 469-475, 2006.

[11] J. Shi, and J. Malik, "Normalized cuts and image segmentation," IEEE Transaction on Pattern Analysis and Machine Intelligence, vol. 22, no. 8,pp. 888-905, 2000.

[12] Gillet, L. Macaire, C. Botte-Lecocq, and J. G. Postaire, "Color image segmentation by analysis of 3D histogram with fuzzy Morphological filters," in Springer-Verlag Editor, Fuzzy Filters for Image Processing-Studies in Fuzziness and Fuzziness and Soft Computing, New York,USA, 2002, pp. 154-177.

[13] C.-C. Cheng, C.-T. Li, and L.-G Chen, "A novel 2D-to-3D conversionsystem using edge information," IEEE Trans. Consumer Electron., vol.56, no.3, pp. 1739-1745, Aug. 2010.

[14] J. Lee, D.-K.Lee, and R.-H. Park, "Robust exemplar-based inpaintingalgorithm using region segmentation," IEEE Trans. Consumer Electron.,vol.58, no. 2, pp. 553-561, May 2012.

[15] S.-G. Jeong, C. Lee, and C.S. Kim, "Motion-compensated frame interpolation based on multi hypothesis motion estimation and texture optimization," IEEE Trans., Image Processing, vol. 22, no. 11, pp.4497-4509, Nov. 2013.

[16] H. Tang and Z. Zhu, "Content-based 3-D mosaics for representing videos of dynamic urban scenes." IEEE Trans., Circuits and Systems forVideo Techno. vol. 22, no. 2, pp. 295-308, Feb. 2012.

[17] K. Fukunaga and L. Hostetler. The estimation of the gradientof a density function, with applications in pattern recognition.IEEE Trans. Information Theory, 21(1):32-40, Jan.1975.

[18] R. Collins. Mean-shift blob tracking through scale space. In'I Computer and Pattern Recognition, pages $234240,2003$.

[19] Z. Zivkovic and B. Krose. An EM-like algorithm for histogram-based object tracking. In Proc. Int'l Conf. Computer Vision and Pattern Recognition,pages 798-803,2004.

[20] B. Han, D. Comaniciu, Y. Zhu, and L. Davis. Incremental density approximation and kernel-based Bayesian filtering for object tracking. In Proc. Int'l Conf. Computer vision and Pattern Recognition, pages 638-644, 2004.

[21] D. Commaniciu, "Mean Shift: A Robust Approach toward Feature Space Analysis," IEEE Transactions on Pattern Analysis and Machine Intelligence, vol. 24, no.5, pp. 603-619, May 2002.

[22] D. Comaniciu, V. Ramesh, and P. Meer. The variable bandwidth mean shift and data-driven scale selection. In Proc. Intel Conf.Computer Vision, pages 498-445, 2001.

[23] H. Chen and P. Meer. Robust fusion of uncertain information.In Proc. Int'l Computer Vision and PatternRecognition, pages 1622,2003.

[24] B.Georgescu, I. Shimshoni and P. Meer. Mean shift based clustering in high dimensions: A texture classification example.In Proc. IEEE Int'1 Computer Vision, pages 456-463, 2003.

[25] D. Comaniciu and P. Meer. Distribution free decomposition of multivariate data. Pattern Analysis and Applications, Springer, 2(1): 22-30; April 1999.

[26] D. Dementhon. Spatial-temporal segmentation of video by hierarchical mean shift analysis. In Proc. Statistical Methods in Video Processing Workshop, 2002.

[27] Dor in Comaniciu and Peter Meer, "Mean shift analysis and applications," in ICCV (2), 1999, pp. 1197-1203.

[28] DeMenthon D., "Spatio-temporal segmentation of video by hierarchical mean shift analysis," in In Proc. Statistical Methods in Video Processing Workshop, Washington, DC, USA, 2002, IEEE Computer Society.

[29] Changjiang Yang, Ramani Duraiswami, Nail A. Gumerov, and Larry Davis, "Improved fast gauss transform and efficient kernel density estimation," in ICCV '03: Proceedings of the Ninth IEEE International Conference on Computer Vision, Washington,DC, USA, 2003, p. 464, IEEE Computer Society.

[30] Kai Zhang, Ming Tang, and J.T. Kwok, "Applying neighborhood consistency for fast clustering and kernel densityestimation," in Computer Vision and Pattern Recognition, IEEE Computer Society Conference on Volume 2,20-25 June, Washington, DC, USA, 2005, pp. 1001 -1007, IEEE Computer Society.

[31] Zhang Y. Tao W., Jin H., "Color imag e segmentation based on mean shift and normalized cuts," in Systems, Man and Cybernetics- Part B, IEEE Transactions on Volume 37, Issue 5, October, Washington, DC, USA, 2007, pp. 1382-1389, IEEE Computer Society.

[32] B. Silverman. Density Estimation for Statistics and Data Analysis. Chapman and Hall, 1986.

[33] C. M. Christoudias, B. Georgescu, and P. Meer, "Synergism in low level vision," in Proc. IEEE Int. Conf. Pattern

[34] Recognition, Quebec City, Canada, vol. 4, pp. 150-155, Aug. 2002. 


\section{Biographies of Authors}

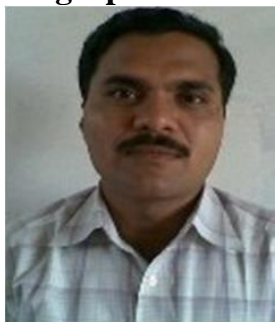

P.Pedda Sadhu Naik received B.Tech (CSE) Degree from JNTUniversity in 2003 and M.Tech (CS) Degree from JNTUCE Anatapur in 2007.He is doing Ph D from JNTUK, Kakinada in Computer Science under the guidance of Dr T. Venu Gopal. He has 12 years of teaching experience. He joined as Assistant Professor in Dr.Samuel George Institute of Engineering \& Technology, Markapur, India in 2003. Presently he is working as Associate Professor and Head of the Department of CSE. His Interested research areas are Image Processing and Soft Computing. He has life member of ISTE and IAENG. He organized various National level Technical Symposiums as Convener. He attended Various National and International Workshops and Conferences on image Processing.

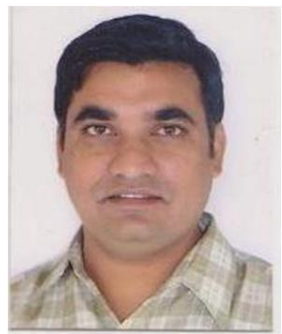

Dr.T.Venu Gopal received B.Tech (ECE) Degree from Osmania University Hyderabad in 1994 and M.Tech (CSE) Degree from JNTUH Hyderabad in 2003.He completed Ph D from JNTUH, Hyderabad in Computer Science. He is Associate Professor, JNTUH College of Engineering, Jagityal. arimnagar District. He has 15years of teaching experience. He has worked in different colleges in different levels. Presently he is working as Associate Professor in JNTUHCE Jagityala. His Interested research areas are Image Processing and Soft Computing. He has life member of ISTE and IAENG. He published more than 30 international journals. He organized various National level Technical Symposiums as Convener. He attended Various National and International Workshops and Conferences on image Processing.

APTIKOM J. CSIT Vol. 1, No. 2, 2016: 46-56 